
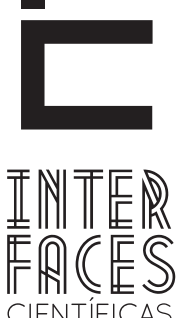

SAÚDE E AMBIENTE

\title{
CÂNCER PEDIÍTRICO: INCIDÊNCIA, SOBREVIDA E MORTALIDADE EM SERGIPE
}

\author{
Margareth Rose Uchoa Rangel ${ }^{1}$ \\ Carlos Anselmo Lima $^{3}$ \\ Rosana Cipolotti ${ }^{5}$

\section{RESUMO}

O principal propósito dos registros de câncer de base populacional é avaliar o impacto do câncer em uma determinada população. Conhecendo a epidemiologia do câncer em determinada região, é possível determinar a necessidade de campanhas de prevenção, implantação de novos métodos diagnósticos, unidades de tratamento e reabilitação. 0 presente trabalho teve como objetivo analisar e apresentar os dados referentes à incidência, sobrevida e mortalidade de 239 casos de câncer em pacientes de zero a 19 anos cadastrados no RCBP de Aracaju, Sergipe, Brasil no período de 1996-2004. Metodologia: Trata-se de um estudo quantitativo, descritivo e retrospectivo. Fontes de dados: Registro de Câncer de Base Populacional (RCBP) de Aracaju, Registro de Óbitos de Sergipe, Instituto Brasileiro de Geografia e Estatística (IBGE), Ministério da Saúde/INCA/Conprev/Divisão de Informação e a População Padrão Mundial, modificada por Doll et
Amaury Lelis dal Fabro ${ }^{2}$

Arthur Rangel Azevedo 4 al (1960). Para o cálculo da probabilidade de sobrevida foi utilizado o método de Kaplan-Meier no programa SPSS versão 19.0 com intervalo de confiança (IC) de $95 \%$ e $p<0,05$. Resultados e conclusões: As taxas ajustadas de incidência foram de 116,3 por milhão de habitantes para o gênero feminino e 142,1 no masculino. A sobrevida em cinco anos foi de $56,9 \%$, sendo maior no grupo dos 5-9 anos, nos pacientes considerados de etnia branca, no gênero feminino e nos tumores sólidos. Melhorou ao longo dos anos, passando de $45,8 \%$ em 1996 para $60,0 \%$ em 2000 e $62,2 \%$ em 2004. A Taxa de mortalidade foi de 69,0 por milhão de habitantes no período estudado.

\section{PALAVRAS CHAVES}

Câncer Pediátrico. Incidência. Sobrevida. Mortalidade. 


\section{ABSTRACT}

The main purpose of the records of population-based cancer is to evaluate the impact of cancer in a given population. Knowing the epidemiology of cancer in a giver region is possible to determine the need for prevention campaigns, implantation of new diagnostic methods, care units and rehabilitation. This study aimed to analyze and present data referring to the incidence, survival and mortality of 239 cases of cancer in patients aged 0 to 19 years registered in the Population-Based Cancer Records in Aracaju, Sergipe, Brazil, from 1996 to 2004. Methodology: This study is quantitative, descriptive and retrospective. The data sources utilized were Population-Based Cancer Records (RCBP) of Aracaju, Sergipe Mortality Records, Brazilian Institute of Geography and Statistics (IBGE), Ministry of Health/INCA/Conprev/Information Division and World Standard Population modified by Doll et al (1960). The survival probability was calculated with the Kaplan-Meier method in SPSS program version 19.0, with confidence interval of $95 \%$ and $p<0.05$. Results and conclusions: The adjusted incidence was 116.3 per million female and 142.1 per million male habitants. The five-year survival rate was $56.6 \%$, being higher in the group of 5-9 years, in patients considered caucasians, in females and in solid tumors. Improved over the years, from 45.8\% in 1996 to $60 \%$ in 2000 and $62.2 \%$ in 2004 . The Mortality Rate was 69 per million habitants in the period studied.

\section{KEYWORDS}

Pediatric Cancer. Incidence. Survival. Mortality.

\section{RESUMEN}

Introducción y objetivos: El principal propósito de los registros de casos cáncer en las poblaciones es avaluar el impacto del cáncer en una determinada población. Conociendo la epidemiologia del cáncer en determinada región, es posible determinar la necesidad de campañas de prevención, implantación de nuevos métodos de diagnóstico, unidades de tratamiento y rehabilitación. El objetivo del presente trabajo fue analizar y presentar los datos referentes a la incidencia, sobrevida y mortalidad en 239 casos de cáncer en pacientes de 0 a 19 años registrados en el RCBP de Aracaju, Sergipe, Brasil en el período de 1996-2004 .Metodología: Este es un estudio cuantitativo, descriptivo y retrospectivo. Fuentes de datos: Registro de Cáncer con Base en la Población (RCBP) de Aracaju, Registro de Muertes en Sergipe, Instituto Brasileiro de Geografía y Estadística (IBGE), Ministerio de la Salud / INCA / Conprev / División de Información y la Población Padrón Mundial, modificada por Doll et al (1960). Para el cálculo de la probabilidad de sobrevida fue utilizado el método de Kaplan-Meier en el programa SPSS versión 19.0 con intervalo de confianza (IC) de $95 \%$ e $p<0,05$. Resultados y conclusiones: Las tasas de incidencia ajustadas fueron de 116,3 por millón de habitantes para el género femenino y 142,1 para el masculino. La sobrevida en cinco años fue de $56,9 \%$, siendo mayor en el grupo de los 5-9 años, en pacientes considerados de etnia blanca, del género femenino y en los tumores sólidos. Al pasar de los años, estas tasas han mejorado, pasando de 45,8\% en 1996 para $60,0 \%$ en 2000 y $62,2 \%$ en 2004 . La tasa de mortalidad registrada en el período estudiado fue de 69,0 por millón de habitantes.

\section{PALABRAS CLAVES}

Cáncer Pediátrico. Incidencia. Sobrevida. Mortalidad. 


\section{INTRODUÇ̃̃̃O}

Apesar de corresponder a apenas 1 a 3\% de todos os diagnósticos de câncer, a cada ano 175.000 crianças no mundo são diagnosticadas com câncer, das quais se estima que 90.000 morram em decorrência da doença. 0 número exato de novos casos não é conhecido, porque em muitos países existe sub-registro e muitas não são diagnosticadas corretamente. Dado alarmante, pois $70 \%$ delas poderiam ser curadas se diagnosticadas e tratadas precocemente. No Brasil, em 2005, a mortalidade de crianças e adolescentes com idade entre 1 e 19 anos correspondeu a $8 \%$ de todos os óbitos, colocando-se, assim como a segunda causa de morte nesta faixa etária, atrás apenas das causas externas ou a primeira causa de morte por doença, trazendo novos desafios para a atenção oncológica e ao Sistema Unificado de Saúde (SUS).

Para descrever a epidemiologia do câncer em uma população é necessário conhecer sua incidência, mortalidade e sobrevida. A incidência é conhecida pelos registros de câncer de base populacional, que através de um processo contínuo e sistemático, de coleta de dados, registra todos os casos novos de câncer que ocorram em uma determinada população de uma área geográfica definida. A mortalidade é conhecida utilizando-se as informações dos bancos de dados de registros vitais. A sobrevida pode ser estudada por meio dos registros populacionais, registros hospitalares e estudos clínicos controlados. Os estudos clínicos consistem no "padrão ouro" para avaliar o trata-

\section{METODOLOGIA}

Trata-se de um estudo quantitativo, descritivo e retrospectivo.

Caracterização da área de estudo: 0 estado de Sergipe é o menor estado brasileiro, está localizado na mento e junto aos registros hospitalares, representam somente uma parte da população selecionada, pois dependem de padrões de encaminhamento hospitalar e critérios de legibilidade dos pacientes. Os estudos baseados em registros populacionais são essenciais para medir o impacto do tratamento e do sistema de saúde do país ou região.

Os registros de câncer com base populacional coletam dados de uma população claramente específica (com diagnóstico de câncer) em uma área geográfica delimitada. São registros que fornecem informações permanentes sobre o número de casos novos nessa área, permitindo detectar setores da área onde a população local é mais afetada pela doença, fatores ambientais que podem estar relacionados e influenciar na prevalência da doença, identificar grupos étnicos afetados promovendo assim investigações epidemiológicas e estudos específicos. As informações obtidas desses registros, também auxiliam na determinação da necessidade de campanhas e prevenção do câncer, como também de novas técnicas diagnósticas. 0 principal propósito deste tipo de registro é avaliar o impacto do câncer em uma determinada população.

0 presente trabalho tem como objetivo analisar e apresentar os dados referentes à incidência, sobrevida e mortalidade em pacientes de 0-19 anos, cadastrados no RCBP de Aracaju, Sergipe, Brasil no período de 1996-2004. 
sendo $36,74 \%$ de zero a 19 anos, segundo o CENSO do IBGE de 2010.

O Registro de Câncer de Base Populacional (RCBP) de Aracaju foi instalado em 1998, no Centro de Oncologia Dr. Osvaldo Leite do Hospital Governador João Alves Filho, registra os casos de câncer incidentes por busca ativa e conta com 10 fontes catalogadas. Estão incluídos todos os laboratórios de patologia e os dois Centros de Alta Complexidade em Oncologia do Estado, o centro de oncologia do Hospital Governador João Alves Filho (HGJAF) e da Fundação Beneficente Hospital de Cirurgia (FBHC), bem como o Sistema de Informação de Mortalidade da Secretaria de Estado da Saúde e o Sistema de Autorização para Procedimentos de Alta Complexidade em Oncologia.

\subsection{MÉTODO:}

A incidência e mortalidade são demonstradas por distribuição percentual (número total de eventos de uma determinada topografia sobre o número total de casos $\times 100$ ), a taxa bruta pelo quoeficiente entre 0 total de eventos e a população sob risco,em um determinado período x 1.000 .000 e a taxa ajustada por idade ou específica é o resultado da multiplicação da taxa específica por idade x população mundial padrão dividida pelo somatório da população mundial.

\section{RESULTADOS}

Foram analisados 239 casos de câncer em pacientes de zero a 19 anos, cadastrados no RCBP de Aracaju-SE, no período de 1996-2004. As fontes dos dados foram os registros hospitalares dos serviços de oncologia da FBHC com 15 casos (6,3\%), do HGJAF com 113 casos (47,3\%), da Onco-hematos com 2 casos $(0,8 \%)$ e da Associação de Voluntários a Serviço da Oncologia em Sergipe (AVOSOS) com 10 casos (4,2\%); Os laboratórios de patologia do estado de Sergipe com 77 casos (29,8\%) e o Sistema de Mortalidade do Estado com 17 casos (7,1\%). 0 número de novos casos por ano variou de 17 (7,1\%; IC 4,2-10,9\%) em 2001, até 38 novos casos (15,9\%; IC 11,3-20,5\%) em 1999. Não houve aumento progressivo ao longo dos anos.
Para o cálculo da probabilidade de sobrevida foi utilizado o método de Kaplan-Meier no programa SPSS versão 19.0 com intervalo de confiança (IC) de $95 \%$ e $p<0,05$. Não foram consideradas sobrevidas quando o $\mathrm{N}$ (número de casos) foi menor que vinte.

0 grupo etário que apresentou maior número de registros foi o do $15-19$ anos com 84 caos (35,1\%; IC $29,3-41,8 \%$ ), seguido pelo grupo do zero aos quatro anos com 58 casos (24,3\%; IC 18,8-29,7\%), dos 10 14 anos com 52 caos (21,8\%; IC 16,7-27,6\%), e por fim, o dos 5-9 anos com 45 (18,8\%; IC 13,8-23,8\%). Os pacientes tiveram seus diagnósticos confirmados por exame histopatológico em 176 casos $(73,6 \%)$ e 32 por citologia $(13,4 \%)$. Os pacientes considerados de etnia branca corresponderam a 28,9\% (IC 23,0$34,7 \%$ ) e os não brancos $71,1 \%$ (IC 63,3-77,0\%). Foram cadastrados 128 pacientes do gênero masculino (53,6\%; IC 46,9-59,8\%) e 111 do feminino (46,4\%; IC 40,2-53,1\%). Os tumores sólidos compreenderam 162 casos (67,8\%; IC 61,5-73,6\%) dos cadastros, enquanto que os hematológicos 77 casos $32,2 \%$; IC 
26,4-38,5\%). As maiores frequências por grupos morfológicos foram: Tumores do sistema nervoso central (SNC) 42 casos (17,6\%; IC 12,6-22,2\%), linfomas 40 casos (16,7\%; IC 11,7-20,1\%), Leucemias 37 casos (15,5\%; IC 11,3-20,1\%) e as menores frequências nos tumores hepáticos 4 casos (1,7\%; IC 0,4-3,3\%), retinoblastomas 5 casos (2,1\%; IC 0,4-4,2\%) tumores do sistema nervoso simpático (SNS) 7 casos (2,9\%; IC 0,8-5,0\%) (Gráfico1)

A taxa incidência ajustada por idade ( $T x A$ ) foi de 116,3 por milhão para os pacientes do gênero feminino e de 142,1 por milhão de habitantes para o masculino. As maiores TxA de incidências foram observadas nos tumores do SNC (30,6/milhão), leucemias (29,0/milhão) e linfomas (25,8/milhão) nos meninos e de 23,4 /milhão nos tumores epiteliais, 17,2 /milhão nas leucemias e 15,5/milhão nos tumores do SNC nas meninas. As menores TxA foram observadas nos retinoblastomas (1,4/milhão), tumores hepáticos (1,4/ milhão) e gonadais (3,2/milhão) nos meninos e de 2,3/milhão nos tumores hepáticos, 3,9 nos tumores do SNS e 5,5 nos retinoblastomas em meninas. As TxA de incidências se mantiveram constantes, ao longo do tempo, sem alterações estatisticamente significativas (Tabela 1)

A probabilidade de sobrevida global em cinco anos foi de $56,9 \%$ (IC 95\% 46,1-67,7\%) no período de 1996-2004 (Gráfico 2). Foi maior no grupo etário de cinco aos nove anos (62,2\%; IC 47,8-76,6\%), seguida pelo grupo de $15-19$ anos (56,9\%; IC 46,3-67,5\%), 10 14 anos (55,8\%; IC 42,0-69,6\%) e de 53,4\% (IC 40,4$66,4 \%)$ no grupo de zero a quatro anos $(p=0,865)$. Os pacientes considerados de etnia branca obtiveram maior sobrevida (62,3\%; IC 51,1-73,5\%) que os não brancos (54,7\%; IC 49,3-64,5\%) ( $p=0,132)$. O gênero feminino obteve maior sobrevida (58,6\%; IC 49,4$67,8 \%$ ) que o masculino (55,5\%; IC 46,7-64,3\%) $(p=0,507)$. Os pacientes com tumores sólidos apresentaram maior sobrevida (58,0\%; IC 50,4-65,6\%) que os com tumores hematológicos (54,5\%; IC 43,1$65,9 \%)(p=0,439)$.

Ao longo dos anos estudados, houve melhora na sobrevida passando de 45,8\% (IC 25,4-66,2\%) no ano de 1996 para $60,0 \%$ (IC 38,0-82,0\%) em 2000 e $62,2 \%$ (IC 46,2-78,2\%) em 2004 ( $p=0,889$ ). Só foi possível analisar a sobrevida por grupos morfológicos tumores do SNC (35,7\%; IC 23,1-53,1\%), nas leucemias e linfomas que foram tumores com alta incidência tanto no sexo masculino como no feminino, apresentaram sobrevida de $51,4 \%$ (IC 35,2-67,5\%) e $57,5 \%$ ( IC 42,3$73,1 \%)$ respectivamente.

A Taxa de mortalidade ajustada por idade e padronizada pela população mundial para o período de 1986-2004 para crianças e adolescentes de 0-19 anos, portadoras de câncer cadastradas no RCBP foi de 69,0 por milhão de habitantes. E não houve modificação expressiva ao longo dos anos.

ted Chilhood Cancer Information (ACCIS), na Europa a taxa de inciência ajustada foi de 140/milhão para crianças de 0-14 anos e de 157/milhão de 15-19 anos. Em 2004 o RCBP de São Paulo apresentou dados de incidência de câncer em menores de 15 anos, sendo de 222,47/milhão para o gênero masculino e 195,03/ milhão para o feminino. Neste trabalho, a taxa de inci- 
dência ajustada foi de 116,3/milhão para o gênero feminino e de 142,1/milhão para o masculino. Sugerindo a existência de sub-registro de câncer em Aracaju.

A incidência específica varia de acordo com o tipo de câncer e com o país ou a região que se estude. $\mathrm{Na}$ América latina os tumores mais frequentes são leucemias, linfomas e tumores de sistema nervoso central; na África predominam os linfomas e na América do Norte os tumores do sistema nervoso central ocupam o segundo lugar, depois das leucemias, que representam 30 a $40 \%$ de todas as neoplasias das crianças, na maioria dos países estudados. Em Aracaju, as maiores incidências foram observadas nos tumores do SNC (30,6/milhão) em meninos e nas neoplasias epiteliais (23,4/milhão) nas meninas, enquanto que as leucemias (29,0/milhão) e os linfomas (25,8/milhão) ocuparam os segundo e terceiros lugares respectivamente nos meninos, e segundo (17,2/milhão) e quarto $(13,5 /$ milhão) lugares nas meninas respectivamente. Fazendo crer que alguns pacientes tenham morrido de leucemias e linfomas sem confirmação diagnóstica, portanto, não foram incluídos nos registros.

As taxas de sobrevida (TS) são indicadores importantes para a avaliação da qualidade dos cuidados prestados à criança com câncer. As maiores taxas de sobrevidas são observadas em países desenvolvidos que possuem centros especializados, técnicas apuradas de diagnósticos com exames radiológicos, de medicina nuclear, biologia molecular e exames citogenéticos, onde os pacientes são submetidos a tratamento segundo protocolos estruturados e em cujas instalações existam condições favoráveis e equipes multiprofissionais especializadas em oncologia.

As taxas de sobrevidas no Canadá (82\%), Europa (75\%) e Estados Unidos (79\%), são exemplos disso. Embora na Europa exista uma grande variação da sobrevida global em cinco anos de acordo com o país estudado, variando desde 90,1\% na Islândia, 81,2\% Finlândia, $81 \%$ Suíça, até 44,9\% na Estônia, sendo os piores resultados observados nos países do leste europeu (65\%). Apesar dos grandes avanços tecnológicos e científicos na abordagem do câncer pediátrico nos países em desenvolvimento, a probabilidade de sobrevida é muito baixa, podendo não passar dos 20 a $25 \%$ em algumas localidades do leste europeu, 37,5\% em Bangalore, na Índia, 37\% em Namíbia, na África; $48 \%$ em Cuba e $48 \%$ em Goiânia, no Brasil.

Neste trabalho, observou-se uma sobrevida global em cinco anos de $56,9 \%$. Sabe-se que diversos fatores podem interferir nas probabilidades de sobreviver ao câncer, como a demora no diagnóstico e no acesso ao centro especializado, levando a estágios avançados da doença, bem como, a falta de adesão ao tratamento, em virtude dos efeitos colaterais dos esquemas terapêuticos reduzindo as chances de sucesso no tratamento. Pode-se considerar que alguns fatores estejam influenciando nos resultados encontrados, como a precariedade de instalações nos serviços de oncologia de Aracaju, a limitação no número de leitos, a falta de continuidade no tratamento, as condições nutricionais dos pacientes, a falta de isolamentos e de leitos em unidade de terapia intensiva, falta de exames diagnósticos, como exames de imagem, laboratoriais, de citogenética e molecular.

Diversos fatores são considerados determinantes no prognóstico e consequentemente na sobrevida dos pacientes pediátricos oncológicos, como: a idade e o estadiamento ao diagnóstico, o tipo histológico, perfil imunológico e genético entre outros.

0 grupo das leucemias, que tem sobrevida de $82,1 \%$ nos EUA no Canadá de $82 \%$. No RCBP de Aracaju a sobrevida nos pacientes com leucemia foi de $51,4 \%$. Nos países em desenvolvimento, assim como na maioria dos estados brasileiros estudados, a sobrevida no grupo das leucemias costuma ser baixa: $31 \%$ em Belo Horizonte, $48 \%$ em Goiânia, $49 \%$ em Fortaleza. Em localidades cujos centros de Oncologia estão melhor estruturados, observa-se uma maior sobrevida destes pacientes, como em Recife com 63\% e São Paulo com $70 \%$. 
Não foi possível analisar as sobrevidas por grupos morfológicos, pois o número de registros era inferior a vinte casos, inviabilizando a análise estatística, o que nos faz crer que exista sub-registro de câncer pediátrico em Aracaju. 0 desenvolvimento e padronização de registros de câncer de base populacional com definição de critérios de qualidade e confiabilidade são pré-requisitos essenciais para realização comparações internacionais.

A taxa de mortalidade observada nesse estudo de 69/milhão de habitantes é extremamente elevada se comparada com taxas observadas no mesmo período nos Estados Unidos (26,9/milhão), Japão (22,0/milhão) e União Europeia (38,8/milhão). No entanto aproxima-se da taxa observada no México (65,0/milhão). É importante ressaltar que a diminuição das taxas de mortalidade nos países desenvolvidos é resultado dos avanços em prevenção, diagnóstico precoce e tratamento adequado em centros especializados.

Os Registros de Câncer de Base Populacional são sistemas de informação que podem fornecer meios de avaliação dos padrões seculares de incidência de câncer na infância. 0 principal objetivo de um RCBP é coletar $100 \%$ dos casos a fim de produzir estatísticas de ocorrências de câncer em uma população definida. A boa qualidade desses dados é fundamental para produzir estatísticas confiáveis para se estabelecer e controlar o impacto que o câncer representa. O RCBP de Aracaju precisa melhorar a quantidade e qualidade dos dados coletados para se tornar representativo da realidade da oncologia pediátrica no estado de Sergipe.

\section{CONCLUSÕES}

No RCBP de Aracaju, no período de 1996-2004, na faixa etária de 0-19 anos, as taxas ajustadas de incidência foram de 116,3 por milhão de habitantes para o gênero feminino e 142,1 no masculino. Em meninos as maiores incidências ocorreram nos tumores do SNC, leucemias e linfomas; as menores incidências, no retinoblastoma, tumores hepáticos e gonadais. Enquanto que nas meninas as neoplasias epiteliais, leucemias e tumores do SNC tiveram as maiores incidências e as menores incidências nos tumores hepáticos, do SNS e retinoblastomas.
A sobrevida em cinco anos foi de $56,9 \%$, sendo maior no grupo dos 5-9 anos, nos pacientes considerados de etnia branca, no gênero feminino e nos tumores sólidos. Melhorou ao longo dos anos, chegando a $62,2 \%$ em 2004.

A Taxa de mortalidade foi de 69,0 por milhão de habitantes no período estudado.

\section{REFERÊNCIAS}

Braga PE, Latorre MRD, Curado MP. Childhood cancer: a comparative analysis of incidence, mortality, and survival in Goiania (Brazil) and other countries. Cad Saude Publica, 2002. 
Boschmonar MG, Alvarez YG, Garcia AM, Soto TN, Roger MC. Childhood cancer survival in Cuba. Eur J Epidemiol, 2000. 16(8): 763-7.

Cantón SBF, Villasenor GP. La mortalidad por tumores malignos en menores de 20 años em México, 19902008. Bol Med Hosp Infantil Mex, 2010. 67: 177-80.

Cha ES, Kong KA, Moon EK, Khang YH, Lee WJ. Childhood Cancer Mortality and Birth Characteristics in Korea: A National Population-based Birth Cohort Study. The Korean Academy of Medical Sciences, 2011. 26: 339-45.

Dama E, Pastore G, Mosso ML, Maule MM, Zuccofo L. Late deaths among five-year survivors of childhood cancer. A population-based study in Piedmont Region, Italy. Haematologica, 91(8): 1084-91, 2006.

Ellison LF, Pogany L, Mery LS. Childhood and adolescent cancer survival: a period analysis of data from the Canadian Cancer Registry. Eur J Cancer, 43(13): 1967-75, 2007.

Ferreira JMO. Incidência, mortalidade e sobrevida de leucemia e linfoma no município de Fortaleza, Ceará [tese]. Rio de Janeiro: Fundação Osvaldo Cruz, 2010.

Gatta, G, Capocaccia R, Coleman MP, Ries GLA, Berrino F. Childhood cancer survival in Europe and the United States. Cancer, 95(8): 1767-72, 2002.

Gatta G, Capacaccia R, Stiller C, Kaatsch P, Terenziani M. Childhood cancer survival trends in Europe: a EUROCARE Working Group study. J Clin Oncol, 23(16): 3742-51, 2005.

Gutierrez AF. Mortalidade por cancer en niños. Bol. Med. Hosp. Infantil México, 62: 1-3, 2005.

Holner MJ, Ries LAG, Krapcho M, Neyman N, Aminou R. SEER Cancer Statistic Review, 1975-2006. Nacional cancer Institute. Bethesda, MD, http://seer.cancer.gov/csr/1975_2006/, based on November 2008 SEER data submission, posted to the SEER web site, 2009.

INCA, SOBOPE. Câncer na criança e no adolescente no Brasil: dados dos Registros de Base Polulacional e de mortalidade. INCA, Ministério da Saúde, Sociedade Brasileira de Oncologia Pediátrica. Rio de Janeiro: Gráfica Esdeva, 220, 2008.

Kaatsch P. Epidemiology of childhood cancer. Cancer Treatment Reviews, 36: 277-85, 2010.

Lacour B, Desandes E, Mallol N, Sommelet D. Lorraine childhood cancer registry: incidence, survival 19831999. Arch Pediatr, 12(11): 1577-86, 2005.

Li J, Thompson TD, Miller JW, Pollack LA,Stewart SL. Cancer incidence among children and adolescents in United States, 2001-2003. Pediatrics;121(6):1470-7, 2008.

Mirra AP, Latorre MRDO, Veneziano BD, editors. Incidência, mortalidade e sobrevida do câncer da infância no município de São Paulo. São Paulo: Registro de Câncer de São Paulo, 2004. 
Pedrosa MF, Pedrosa F, Lins MM, Pontes Neto NT, Hanois filho G. Non-Hodgkin's lymphoma in childhood: clinical and epidemiological characteristics and survival analysis at a single center in Northeast Brazil. J Pediatr (Rio J), 83(6): 547-54, 2007.

Rangel MRU, Santos KSSJ, Cipolotti R., Fontes AM. Epidemiologia do Câncer Pediátrico no Estado de Sergipe-Brasil. Atualização e Estudo Comparativo. SIICSalud 2006; 1-9 DOI. Disponível em: <http://www.siicsalud. com/dat049/06802015.htm>. Acesso em: 05/04/2013.

Ries LAG, Eisner MP, Kosary CL, Hankey BF, MillierBA, Clegg L. SEER. Cancer statistic review, 1973-1997 (monography on the internet). Bethesda: National Cancer Institute; 2002 [cited 2008 out 2]. Available from: http:// seer.cancer.gov/csr/1973_1997/.

Steliarova-Foucher, E, Stiller C, Kaatsch P, Berrino F Coeberg JW. Geographical patterns and time trends of cancer incidence and survival among children and adolescents in Europe since the 1970s (the ACCISproject): an epidemiological study. Lancet, 364(9451): 2097-105, 2004.

Viana MB, Cunha KCCMS, Ramos G, Mourão M. Acute myeloide leukemia in childhood: fifteen-year experience in a single instituition. J Pediatr (Rio J), 79(6): 489-96, 2003. ensino da UFS. E:mail: limaca@infonet.com.br

4 Mestrado em Saúde da Criança pela UFS (2000). Professor Auxiliar de ensino da UFS. E-mail: rangel.aaz@gmail.com

5 Doutorado em Oncologia Pediátrica pela Universidade de São Paulo Ribeirão Preto (2000). Professor Adjunto da UFS. E-mail: rosanaci@yahoo.com 


\section{ANEXOS}

Gráfico1 - Distribuição dos tumores por grupos morfológicos, em menores de 20 anos, cadastrados no RCBP de Aracaju-Se, $1996-2004$.

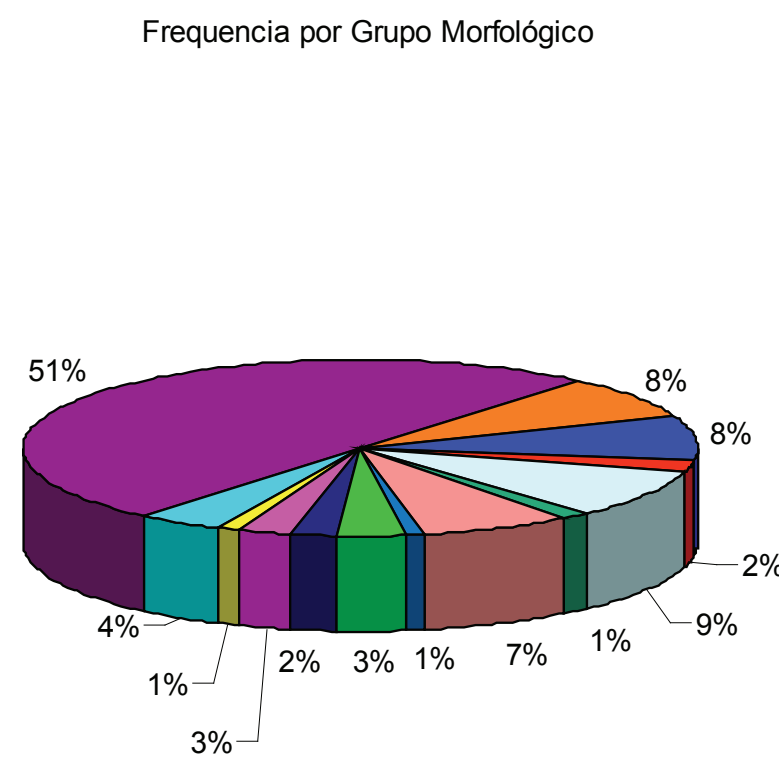

$\square$ Leucemias
$\square$ Linfomas e NRE
$\square$ Neo germ trof gonadais
$\square$ Neoplasias do SNC
$\square$ Neoplasias do SNS
$\square$ Neoplasias epiteliais
$\square$ Neoplasias hepaticas
$\square$ Neoplasias osseas
$\square$ Retinoblastoma
$\square$ O Outras neoplasias malignas não
especificadas
$\square$ Narcoma de partes moles

Tabela 1 - Incidência por subtipos histológicos por gênero em menores de 20 anos, cadastrados no RCBP de Aracaju-Se, $1996-2004$.

\begin{tabular}{|c|c|c|c|c|c|c|c|c|}
\hline \multirow[b]{3}{*}{ GRUPOS MORFOLÓGICOS } & \multicolumn{8}{|c|}{ INCIDÊNCIA } \\
\hline & \multicolumn{3}{|c|}{ Masc } & \multicolumn{5}{|c|}{ Fem } \\
\hline & $\mathbf{N}$ & $\%$ & TxB & TXA & $\mathbf{N}$ & $\%$ & TXB & TXA \\
\hline I- Leucemias & 24 & 18,74 & 26,2 & 29,07 & 14 & 12,61 & 15,14 & 17,25 \\
\hline Ia- Leucemias llinfoides & 17 & 13,28 & 18,54 & 20,85 & 13 & 11,71 & 14,05 & 16,42 \\
\hline $\begin{array}{l}\text { Ib- Leucemias agudas não linfablás- } \\
\text { ticas }\end{array}$ & 3 & 2,34 & 3,28 & 3,9 & 0 & 0 & 0 & 0 \\
\hline Ic- Leucemias mielóides crônicas & 1 & 0,78 & 1,05 & 0,83 & 1 & 0,9 & 1,08 & 0,83 \\
\hline Id- Outras leucemias especificadas & 1 & 0,78 & 1,06 & 1,4 & 0 & 0 & 0 & 0 \\
\hline le- Leucemias não especificadas & 2 & 1,56 & 2,24 & 2,08 & 0 & 0 & 0 & 0 \\
\hline
\end{tabular}




\begin{tabular}{|c|c|c|c|c|c|c|c|c|}
\hline II- Linfomas e neo do SER & 25 & 19,52 & 27,1 & 25,88 & 13 & 11,72 & 13,93 & 13,59 \\
\hline Ila- Linfoma de Hodgkin & 4 & 3,12 & 4,39 & 3,64 & 6 & 5,41 & 6,4 & 5,11 \\
\hline Ilb- Linfomas não Hodgkin & 15 & 11,72 & 16,33 & 15,29 & 6 & 5,41 & 6,48 & 7,15 \\
\hline Ilc- Linfoma de Burkitti & 4 & 3,12 & 4,25 & 4,72 & 1 & 0,9 & 1,05 & 1,32 \\
\hline lle- Linfomas não especificados & 2 & 1,56 & 2,12 & 2,21 & 0 & 0 & 0 & 0 \\
\hline IIl- Tumores do SNC & 27 & 21,1 & 29,47 & 30,65 & 15 & 13,51 & 16,12 & 15,55 \\
\hline Illa- Ependimomas & 1 & 0,78 & 1,11 & 0,87 & 1 & 0,9 & 1,05 & 0,8 \\
\hline lllb- Astrocitomas & 12 & 9,38 & 12,88 & 14,86 & 6 & 5,41 & 6,42 & 6,42 \\
\hline lllc- Tumores neuroectodérmicos & 5 & 3,91 & 5,52 & 5,3 & 3 & 2,7 & 3,21 & 3,31 \\
\hline Illd- Outros Gliomas & 0 & 0 & 0 & 0 & 2 & 1,8 & 2,14 & 1,64 \\
\hline lllf- Outros não especificados & 9 & 7,03 & 9,96 & 9,6 & 3 & 2,7 & 3,28 & 3,36 \\
\hline IV-Tumores do SNS & 3 & 2,43 & 3,26 & 3,69 & 3 & 2,7 & 3,21 & 3,97 \\
\hline $\begin{array}{l}\text { IVa- Neuroblastoma e ganglioneu- } \\
\text { roblastoma }\end{array}$ & 2 & 1,56 & 2,2 & 2,64 & 2 & 1,8 & 2,11 & 3,14 \\
\hline IVb- Outros tumores do SNS & 1 & 0,78 & 1,05 & 1,04 & 1 & 0,9 & 1,1 & 0,82 \\
\hline V- Retinoblastoma & 1 & 0,78 & 1,08 & 1,42 & 4 & 3,6 & 4,27 & 5,54 \\
\hline VL- Tumores renais & 6 & 4,69 & 6,63 & 8,15 & 5 & 4,5 & 5,39 & 6,84 \\
\hline $\begin{array}{l}\text { Vla- Tumor de Wilms, sarc rabd e cel. } \\
\text { Claras }\end{array}$ & 5 & 3,91 & 5,49 & 6,58 & 4 & 3,6 & 4,31 & 5,88 \\
\hline Vlb- Carcinomas & 1 & 0,78 & 1,13 & 1,57 & 1 & 0,9 & 1,08 & 0,95 \\
\hline VIl- Tumores hepáticos & 2 & 1,56 & 2,15 & 1,98 & 2 & 1,8 & 2,16 & 2,33 \\
\hline Vlla- Hepatoblastoma & 1 & 0,78 & 1,09 & 1,21 & 1 & 0,9 & 1,08 & 1,47 \\
\hline Vllb- Hepatocarcinoma & 1 & 0,78 & 1,05 & 0,77 & 1 & 0,9 & 1,08 & 0,85 \\
\hline Vlll- Tumores ósseos & 9 & 7,03 & 9,86 & 9,49 & 8 & 7,2 & 8,55 & 7,16 \\
\hline Vllla- Osteossarcomas & 5 & 3,91 & 5,44 & 4,52 & 5 & 4,5 & 5,32 & 4,61 \\
\hline Vlllb- Condrossarcomas & 1 & 0,78 & 1,08 & 0,95 & 0 & 0 & 0 & 0 \\
\hline Vlllc- Sarcomas de Ewing & 1 & 0,78 & 1,12 & 0,96 & 2 & 1,8 & 2,12 & 1,71 \\
\hline Vllle- Outros tumores ósseos NE & 2 & 1,56 & 2,21 & 3,05 & 1 & 0,9 & 1,1 & 0,84 \\
\hline IX-Sarcomas de partes moles & 13 & 10,15 & 14,19 & 13,96 & 7 & 6,3 & 7,47 & 7,99 \\
\hline IXa-Rabdomiossarcomas & 7 & 5,47 & 7,65 & 8,63 & 2 & 1,8 & 2,12 & 2,63 \\
\hline
\end{tabular}


IXb- Fibrossarcomas e neurofibros-

\begin{tabular}{|c|c|c|c|c|c|c|c|c|}
\hline sarcomas & 0 & 0 & 0 & 0 & 2 & 1,8 & 2,15 & 2,34 \\
\hline IXc- Sarcoma de Kaposi & 0 & 0 & 0 & 0 & 1 & 0,9 & 1,07 & 0,75 \\
\hline IXd- Outros sarcomas & 3 & 2,34 & 3,28 & 2,8 & 1 & 0,9 & 1,05 & 1,2 \\
\hline IXe- Outros sarcomas NE & 3 & 2,34 & 3,25 & 2,52 & 1 & 0,9 & 1,05 & 1,06 \\
\hline $\begin{array}{l}\text { X- Tu. de cel. germinativas, trofo- } \\
\text { blásticas e gonadais }\end{array}$ & 3 & 2,34 & 3,32 & 3,25 & 8 & 7,2 & 8,56 & 9,55 \\
\hline $\begin{array}{l}\text { Xa- Tumores germinais intracrania- } \\
\text { nos e especificados }\end{array}$ & 2 & 1,56 & 2,18 & 2,21 & 0 & 0 & 0 & 0 \\
\hline $\begin{array}{l}\text { Xb- Tumores germinais não gona- } \\
\text { dais }\end{array}$ & 0 & 0 & 0 & 0 & 3 & 2,7 & 3,24 & 3,95 \\
\hline Xc- Tumores germinais gonadais & 0 & 0 & 0 & 0 & 4 & 3,6 & 4,28 & 4,71 \\
\hline Xd-Carcinomas gonadais & 0 & 0 & 0 & 0 & 1 & 0,9 & 1,04 & 0,88 \\
\hline Xe- Tumores gonadais e NE & 1 & 0,78 & 1,13 & 1,04 & 0 & 0 & 0 & 0 \\
\hline Xl- Neoplasias epiteliais & 14 & 10,93 & 15,31 & 13,29 & 29 & 26,13 & 31,01 & 23,48 \\
\hline Xlb- Carcinoma de Tireóide & 4 & 3,12 & 4,41 & 3,7 & 7 & 6,31 & 7,44 & 5,78 \\
\hline Xlc- Carcinoma de nasofaringe & 1 & 0,78 & 1,13 & 1,04 & 0 & 0 & 0 & 0 \\
\hline Xld- Melanoma Malígno & 1 & 0,78 & 1,11 & 1 & 0 & 0 & 0 & 0 \\
\hline Xle- Carcinoma cutâneo & 6 & 4,69 & 6,53 & 5,73 & 12 & 10,81 & 12,8 & 9,71 \\
\hline Xlf- Outros carcinomas e NE & 2 & 1,56 & 2,12 & 1,81 & 10 & 9,01 & 10,76 & 7,99 \\
\hline Xll- Outros tumores malígnos & 1 & 0,78 & 1,13 & 1,27 & 3 & 2,7 & 3,24 & 3,04 \\
\hline TOTAL & 128 & 100 & 139,75 & 142,14 & 111 & 100 & 119,12 & 116,31 \\
\hline
\end{tabular}

Gráfico 2 - Probabilidade de sobrevida global em cinco anos. Curva de Kaplan- Meier.

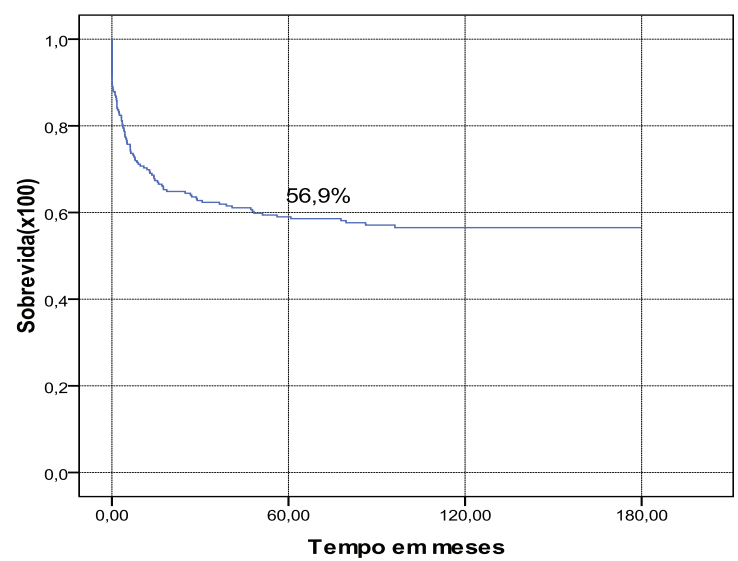

